\title{
Arqueologia da escravidão em fazendas jesuíticas: primeiras notícias da pesquisa*
}

\author{
The archeology of slavery on Jesuit \\ fazendas: first research notes
}

\section{Luís Cláudio P. Symanski}

Professor do Departamento de Sociologia e Antropologia/ Faculdade de Filosofia e Ciências Humanas/Universidade Federal de Minas Gerais.

Av. Antônio Carlos, 6627

31270-901 - Belo Horizonte - MG

- Brasil

luis.symanski@pq.cnpq.br

\section{Flávio Gomes}

Professor dos programas de pósgraduação em Arqueologia e em História Comparada/Universidade Federal do Rio de Janeiro; pesquisador do Conselho Nacional de Desenvolvimento Científico e Tecnológico.

Largo de São Francisco, 1

20050-070 - Rio de Janeiro - RJ Brasil

escravo@prolink.com.br
SYMANSKI, Luís Cláudio; GOMES, Flávio. Arqueologia da escravidão em fazendas jesuíticas: primeiras notícias da pesquisa. História, Ciências, Saúde - Manguinhos, Rio de Janeiro, v.19, supl., dez. 2012, p.309-317.

\section{Resumo}

Nesta nota de pesquisa apresentamos questões teóricas e metodológicas sobre uma investigação em arqueologia histórica iniciada recentemente, que visa analisar o cotidiano da escravidão, regimes demográficos, práticas culturais etc. Um levantamento de sítios arqueológicos em antigas senzalas e fazendas escravistas do Vale do Paraíba e norte fluminense está sendo realizado. Com a cooperação de historiadores, arqueólogos e antropólogos, registros da cultura material de populações escravas de origem indígena e depois africana estão sendo localizados nas escavações iniciadas na fazenda jesuítica do Colégio em Campos dos Goytacazes (RJ), administrada por religiosos e depois leigos nos séculos XVII, XVIII e XIX.

Palavras-chave: arqueologia histórica; escravidão; cultura material; engenhos, Campos dos Goytacazes.

\section{Abstract}

These preliminary research notes present theoretical and methodological questions regarding a recently inaugurated investigation in historical archeology that intends to analyze daily life under slavery, demographic regimes, cultural practices, and so on. A survey of archeological sites on former 'senzalas' (slave quarters) and slave-owning fazendas in the Paraíba Valley and northern part of the state of Rio de Janeiro is currently in progress. With the cooperation of historians, archeologists, and anthropologists, records of the material culture of slave populations, which originally comprised indigenes and later Africans, are being located at excavations underway on the fazenda that is part of the Jesuit school in Campos dos Goytacazes, Rio de Janeiro, first run by the clergy and later by members of the laity in the seventeenth, eighteenth, and nineteenth centuries.

Keywords: historical archeology; slavery; material culture; sugar plantations; Campos dos Goytacazes. 
Tas últimas décadas, os estudos sobre a escravidão no Brasil mobilizaram muitos
pesquisadores, com debates e uso de fontes e perspectivas teóricas e metodológicas diversas. Como resumo das principais características desse movimento historiográfico: estudos acadêmicos, resultantes de teses e dissertações produzidas por pesquisadores treinados em programas de pós-graduação; a diversidade nas perspectivas teórico-metodológicas adotadas, reunindo tanto aspectos multidisciplinares como o diálogo com estudos desenvolvidos em outras regiões das Américas; o uso cada vez mais ampliado e original de métodos quantitativos (Klein, 2009); pesquisas sistemáticas baseadas em fontes primárias de natureza diversa e em diferentes acervos; a diversificação cada vez maior na escolha das temáticas, mas também a revisão de temas clássicos; estudos de caso ou enfoques em regiões específicas; e ênfase nas análises sobre a experiência cotidiana dos escravos e libertos, contemplando as várias visões sobre a escravidão e a liberdade.

É possível identificar mudanças de rumos e ênfase em alguns eixos temáticos e metodológicos. Como exemplo vejamos a atenção voltada para abordagens em torno da ideia de cultura na sociedade escravista. Por muito tempo falou-se genericamente da reminiscência da cultura africana, sendo necessário, para alguns, classificá-la. Foi na direção de uma 'tradição' que determinados trabalhos antropológicos se encaminharam. Guardadas suas especificidades, foram percorridos nomes como Nina Rodrigues (1977), Arthur Ramos (1935, 1942, 1953, 1979), Edison Carneiro (1964) e Roger Bastide (1974, 1985). A própria antropologia demonstrou os caminhos dessa '(re)invenção' da África no Brasil. ${ }^{1}$ Para a crítica de tais abordagens sobre as origens da cultura africana foram fundamentais os estudos clássicos de João Reis (2003) e Robert Slenes (1999), entre outros. As ideias em torno do 'africano' genérico e de uma 'crioulização' a-histórica foram reconfiguradas. De quem falamos? Quais os impactos do tráfico atlântico e as sociedades africanas envolvidas? E os mundos do trabalho, da formação de famílias e das identidades na escravidão nas Américas? Mais ainda: e a cultura material da sociedade escravista?

É justamente esta última questão que envolve os estudos arqueológicos sobre a escravidão no Brasil, sob a premissa de que a arqueologia, como uma ciência social que tem como fonte principal a cultura material, constitui uma via de acesso à história e cultura dos grupos escravos que é simultaneamente alternativa e complementar aos registros escritos. O registro arqueológico desses grupos consiste, basicamente, nos vestígios materializados de suas práticas cotidianas, práticas que, muitas vezes, foram mantidas ocultas das vistas dos segmentos dominantes. Apresenta, assim, um enorme potencial de fornecer informações, não passíveis de serem obtidas por outras fontes, sobre os padrões de vida material, economia, dinâmica social, cosmologias, religiosidade, construção e reconstrução de identidades e agência das populações africanas na diáspora.

Ainda assim, as investigações em sítios de ocupação africana e afrodescendente no Brasil ainda são escassas, de forma diversa do que ocorre nos EUA, onde pesquisas sistemáticas sobre a escravidão vêm sendo realizadas desde o início da década de 1970 em contextos como plantations, chácaras, comunidades de fugitivos e unidades domésticas rurais e urbanas (Fennell, 2011).

O estudo de Guimarães e Lanna (1980) em quilombos de Minas Gerais foi o marco inaugural da arqueologia da escravidão no Brasil, seguido por escavações no quilombo do 
Ambrósio em Minas Gerais (Guimarães et al., 1990). Essas pesquisas, porém, não tiveram continuidade, resultando apenas na publicação dos resultados iniciais. Em 1992 e 1993, Orser e Funari (1932) realizaram escavações exploratórias no quilombo dos Palmares, localizado na Serra da Barriga em Alagoas. ${ }^{2}$ Ainda no começo da década de 1990, foram realizadas escavações na senzala da fazenda São Fernando, em Vassouras (RJ) (Lima, Bruno, Fonseca, 1993). Pesquisas mais recentes incluem escavações em três pequenos quilombos do Rio Grande do Sul (Carle, 2005), novas escavações no quilombo dos Palmares (Allen, 2006), escavações em uma senzala urbana em Martinho da Serra (RS) (Machado, Milder, 2006), em senzalas de dois engenhos de açúcar e em um quilombo da chapada dos Guimarães (MT) (Symanski, 2006; Symanski, Souza, 2006) e nas senzalas do engenho São Joaquim, em Pirenópolis (GO) (Souza, 2011).

Entre outras questões, algumas investigações têm discutido as formas como os escravos - em variados contextos - gestaram uma cultura material visando à manutenção, reprodução e transformação de cosmologias e práticas rituais, conforme evidenciado pela reprodução de escarificações em vasilhames cerâmicos artesanais, pelo uso de possíveis itens devocionais de matriz africana, como os colares dos sepultamentos da Sé de Salvador (Tavares, 2006), um cristal negro encontrado na senzala do engenho São Joaquim, em Goiás, e cristais de quartzo implantados sob o piso da casa-grande do engenho Rio da Casca, em Mato Grosso (Symanski, 2007; Souza, Agostini, no prelo). O processo de trocas culturais dos grupos classificados como africanos, indígenas e europeus tem sido abordado com base nos modelos correlatos de crioulização, transculturação e etnogênese. Crioulização consiste em um processo envolvendo interações e trocas complexas que resultaram em novas formas culturais. Esse modelo busca incluir o efeito da experiência do Novo Mundo sobre todos os grupos populacionais, os euro-americanos (Singleton, 1998; Dawdy, 2000) entre eles. Na arqueologia, o uso desse modelo levou a um esforço para entender a cultura material dos sítios de ocupação africana e afro-americana com base nos referenciais culturais próprios desses grupos, em vez de levar em conta apenas os significados atribuídos pela cultura hegemônica.

O modelo de crioulização, contudo, tem sido criticado por obscurecer a identidade cultural de grupos étnicos específicos e por ser estático, posto que desconsidera o papel ativo dos atores em sua capacidade de manipular mais de um tipo de linguagem, de estilo comportamental ou de repertório material de acordo com os seus interesses (Singleton, 1998; Gundaker, 2000). O que deve ser considerado é que esse processo de emergência de identidades crioulizadas, que anterior ao se assumia até então, deve ser contextualmente avaliado, pois pode apresentar ritmos e impactos distintos de acordo com as especificidades da escravidão em diferentes regiões das Américas, bem como ser marcado por períodos de expressão de diferenças e reconstrução de identidades pautadas em referenciais diversificados, conforme sugerem estudos arqueológicos em contextos do Mato Grosso (Souza, Symanski, 2009).

É nesse âmbito de desenvolvimento dos estudos arqueológicos sobre a escravidão no Brasil que está sendo desenvolvido o projeto 'Café com açúcar: arqueologia da escravidão em uma perspectiva comparativa no sudeste rural escravista, séculos XVIII e XIX '3, que visa investigar a vida material de grupos escravos das plantations do Sudeste, a partir de escavações 
em senzalas coletivas e familiares de dois tipos principais de unidades de produção: engenhos de açúcar e fazendas de café.

A proposta do projeto é adotar uma perspectiva comparativa, com o objetivo de obter informações sobre as diversificadas configurações econômicas, sociais e culturais desenvolvidas pelos grupos escravizados, em função tanto da estrutura produtiva quanto da composição cultural diferenciada desses plantéis. O foco recai, por um lado, nas estratégias de dominação empregadas pelos senhores nesses estabelecimentos e, por outro, nos mecanismos desenvolvidos pelos escravos para lidar com elas, bem como sobre as possibilidades buscadas pelos escravos para a realização de seus próprios projetos nessas estruturas de limitação, que dizem respeito à capacidade de agência desses grupos. O projeto, assim, está orientado para as seguintes questões: houve diferenças nos padrões de vida material, econômica, social e cultural dos grupos escravos em função do tipo de estrutura produtiva? Esses grupos conseguiram agenciar diferentemente suas vidas nesses diferentes contextos? A diversidade da composição cultural dos plantéis levou a diferenças nas configurações materiais e sociais desses grupos? Houve variações nas táticas de resistência desses grupos em função dos tipos diferenciados de estruturas produtivas? Ou a variação cultural dos plantéis teve mais influência nos tipos de táticas desenvolvidos? Puderam os grupos africanos manter elementos de suas culturas? Foi possível a grupos de origem africana diferenciada conformar subgrupos nesses espaços, pautados em afinidades culturais comuns em suas respectivas regiões de origem? E, por fim, qual foi o ritmo do processo de crioulização no Sudeste escravista brasileiro sob diferentes regimes produtivos? Houve amplas regularidades nesse processo, ou as especificidades regionais de ordem econômica e cultural levaram à conformação de culturas crioulas diferenciadas?

Tais questões deverão ser discutidas com base em amplo estudo da cultura material, envolvendo desde o nível macro da configuração espacial das unidades de produção, visando entender as formas como esses espaços foram estruturados e articulados, até a dimensão micro do contexto de deposição de um dado artefato e de suas relações contextuais com outros artefatos do mesmo nível e camada deposicional, bem como com as estruturas do entorno, que incluem desde fogueiras até as edificações das senzalas, casas-grandes e demais benfeitorias. Considera-se que os artefatos produzidos pelos escravos podem ter papel ativo na reconfiguração das identidades desses grupos, pois que permitem a reprodução de estilos que podem ter sido típicos de suas sociedades africanas matrizes. O estudo da diversidade estilística espacial e diacrônica, assim, pode apontar para mecanismos de retenção de estéticas, significados e mesmo cosmologias de diferentes grupos africanos, como também pode informar sobre os processos de mudanças ocasionados pelas trocas culturais entre grupos diversificados (Ferguson, 1992; Souza, Symanski, 2009). O material zooarqueológico, por outro lado, pode informar sobre as práticas de subsistência desses grupos, envolvendo estratégias como a caça, a pesca e a criação de animais para consumo próprio, sobre os hábitos alimentares por eles mantidos, incluindo a preferência por determinadas espécies e o acesso a determinados cortes de carne (Heinrich, 2012; Souza, 2011, p.93). Por fim, o agrupamento de certos itens, intencionalmente enterrados ou encontrados junto a estruturas como fogueiras (tais como crucifixos, moedas perfuradas, cristais, chifres, conchas, pregos, esqueletos de animais etc.), pode apontar para práticas rituais de africanos na diáspora, e, 
desse modo, contribuir para o entendimento do processo histórico da conformação das religiões de matriz africana nos cenários rurais (Wilkie, 1995; Leone, Fry, 2001; Symanski, 2007).

Foram selecionadas duas regiões para os trabalhos de campo: Campos dos Goytacazes, caracterizada pela maciça presença de engenhos de açúcar nos séculos XVIII e XIX, com intenso uso da mão de obra escravizada (Faria, 1998; Lara, 1988); e o vale do Paraíba, região em que conformou, no primeiro quartel do século XX, uma economia cafeeira de dimensões globais, com suntuosas fazendas, tornando-se, em consequência, uma das principais áreas importadoras de africanos do Sudeste (Stein, 1990; Silva, 1984).

A primeira fase das investigações arqueológicas teve início em julho de 2012, na fazenda dos Jesuítas de Campos dos Goytacazes. Sua sede foi construída em meados do século XVII por padres da Companhia de Jesus. Um século mais tarde, a propriedade foi arrematada pelo comerciante Joaquim Vicente dos Reis. A sede da propriedade é uma das mais antigas construções de Campos dos Goytacazes. Tombada pelo Patrimônio Histórico em 1946 e desapropriada pelo governo do estado na década de 1970, resistiu com seu último morador, João Batista Barroso, até 1980, ano de sua morte. A partir de então, o edifício foi abandonado. Em 1991 passou por processo de restauração para que fosse implantada a Escola de Cinema da Universidade Estadual do Norte Fluminense, sofrendo posteriormente novo abandono. Em 2001 foi ocupado pelo Arquivo Público Municipal de Campos dos Goytacazes.

As escavações arqueológicas contemplaram duas áreas: uma de deposição de refugo referente aos ocupantes da sede, situada cerca de $45 \mathrm{~m}$ a noroeste da sede; e a outra $80 \mathrm{~m}$ a norte da sede, referente à extremidade noroeste de uma grande senzala em conformação de $U$ que ficava de frente para a sede, cujas extremidades originalmente ultrapassavam a linha da parede frontal da sede da fazenda em aproximadamente $10 \mathrm{~m}$, formando uma praça de cerca de $180 \mathrm{~m}$ x 180m (Figura 1).

$\mathrm{Na}$ área de deposição de refugo da sede da fazenda foram abertas duas unidades de escavação, totalizando $9 \mathrm{~m}^{2}$ de área escavada, com profundidade média de $40 \mathrm{~cm}$, suficiente para recuperar amostra significativa de material arqueológico referente ao período entre o final do século XVIII e meados do século XIX, incluindo grande quantidade de porcelanas europeias, louças inglesas, restos alimentares, predominando ossos de bois. Na área referente à senzala foram abertas duas unidades de escavação, iniciadas na forma de trincheiras paralelas alinhadas no sentido norte-sul, a $15 \mathrm{~m}$ de distância uma da outra. A trincheira leste, com $9 \mathrm{~m}$ de comprimento, e a trincheira oeste, com $11 \mathrm{~m}$, sendo ambas subdivididas em quadrículas de $1 \mathrm{~m} \times 1 \mathrm{~m}$. Ambas foram rebaixadas até atingir a base do depósito arqueológico, entre 40 e $50 \mathrm{~cm}$ de profundidade. A trincheira oeste apresentou escassa quantidade de material construtivo, ao passo que na trincheira leste foi encontrada grande quantidade desse tipo de material, representado por telhas e tijolos, indicando tratar-se, esta última, da extremidade noroeste da senzala em U. A área de escavação das duas trincheiras foi a seguir expandida, abrindo-se área adicional de $5 \mathrm{~m}$ x $3 \mathrm{~m}$ anexa à trincheira leste, e mais 12 quadrículas de $1 \mathrm{~m}$ x $1 \mathrm{~m}$ no entorno da trincheira oeste.

Verificou-se que ambas as áreas de escavação eram locais de deposição de refugo dos ocupantes da senzala, provavelmente daqueles que habitavam a ala noroeste. Eles tenderam 


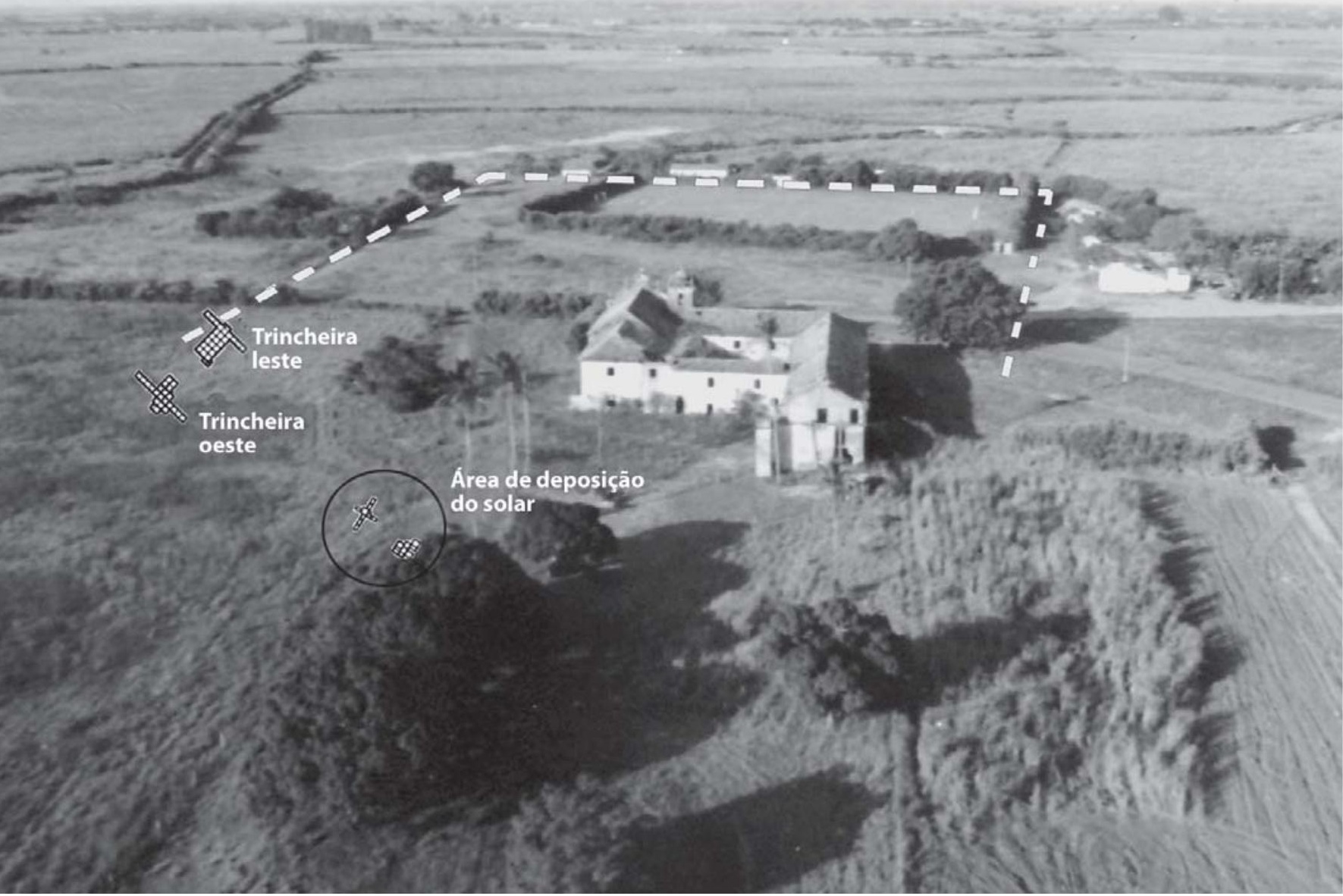

Figura 1: Fotografia aérea do Solar do Colégio em 1980, com indicação das áreas escavadas; o tracejado branco diz respeito ao traçado da senzala (Arquivo Público de Campos dos Goytacazes).

a depositar seu refugo sobretudo além da extremidade da senzala, na área da trincheira oeste. Para tanto, abriram valas irregulares rasas nesse local. Provavelmente como forma de higienização, em geral cobriam o conteúdo dessas valas, representado por uma enorme quantidade de material orgânico - conforme indicado pelos ossos de animais domésticos e silvestres -, com uma camada de telhas, tornando, a seguir, a depositar mais refugo sobre essa camada e, novamente, cobrindo com outra camada de telhas. Já a área da trincheira leste era, provavelmente, referente à última unidade de habitação da senzala. Não foram, contudo, identificados os alicerces dessa edificação, somente, como mencionado, vestígios do material de sua construção. Porém, evidências da estrutura de uma fogueira nessa área, composta por grandes tijolos soltos, em conformação circular, contornando uma vala que continha grande quantidade de carvão, além de material ósseo e mariscos, indicaram que essa área era adjacente à última unidade de habitação da senzala, tendo sido utilizada por seus ocupantes em atividades relacionadas ao preparo e consumo de alimentos.

As duas unidades de escavação referentes à área da senzala apresentaram grande quantidade de material arqueológico, sendo cerca de 50\% da amostra referente a material ósseo de animais domésticos como bois, porcos e galinhas, mas também silvestres e aquáticos, incluindo capivaras, porcos-do-mato e peixes. Entre os itens da cultura material foi recuperada uma grande quantidade de fragmentos de cerâmicas artesanais de produção 
local ou regional, cerâmicas torneadas, faianças portuguesas do século XVIII e louças inglesas do século XIX, ornamentos simples de cobre martelado, como pulseiras e pingentes, contas de colares de vidro e cachimbos de cerâmica. As características desse material apontam para um intervalo de deposição concentrado entre 1800 e 1850, não alcançando, assim, a ocupação setecentista referente ao plantel de escravos dos jesuítas.

A amostra está em processo de análise nas dependências do Centro de Estudos e Pesquisas Arqueológicas da Universidade Federal do Paraná, passando por procedimentos de limpeza, restauração, curadoria, identificação e catalogação. Os resultados da análise trarão diversas informações inéditas sobre a vida cotidiana, os hábitos alimentares, a economia e os sistemas de crenças da população cativa que ocupou a fazenda dos Jesuítas na primeira metade do século XIX. Em uma perspectiva comparativa, essas informações servirão como base para caracterizar a vida cotidiana dessa população, suas similaridades e diferenças com aquela dos grupos que, nessa época, trabalhavam compulsoriamente nas fazendas de café do Vale do Paraíba, região em que será desenvolvida a próxima etapa do projeto.

\section{AGRADECIMENTOS}

Carlos Roberto Freitas, diretor do Arquivo Público Municipal de Campos dos Goytacazes, bem como às funcionárias Rafaela Machado e Larissa Manhães Ferreira pelo total apoio à realização da pesquisa de campo. O sucesso da escavação arqueológica deveu-se ao incansável trabalho da equipe, composta por Bárbara de Ridder Barros, Caetano Tocchetto, Carlos Eduardo Lançoni, Cibele da Rocha, Daniele Jesus, Fernando Cantele, Fernando Myashita, Isabela Suguimatsu, Jean Lovato, Kendra Andrade, Luara Stollmeier, Lucas Roahny da Silva, Monique Seidel, Patrícia Marinho, Sabrina Andrade, Suzana Munsberg e Tamires Lico.

\section{NOTAS}

* Um levantamento de sítios arqueológicos em antigas senzalas e fazendas escravistas do Vale do Paraíba e norte fluminense está sendo financiado pelo Conselho Nacional de Desenvolvimento Científico e Tecnológico (CNPq) e Fundação de Amparo à Pesquisa do Estado do Rio de Janeiro (Faperj), através da Universidade Federal do Rio de Janeiro e da Universidade Federal do Paraná. A primeira etapa dessa pesquisa contou com o financiamento do CNPq e o apoio do Arquivo Público e da Prefeitura Municipal de Campos dos Goytacazes.

${ }^{1}$ Ver Cunha, 1985; Dantas, 1982; Farias, Soares, Gomes, 2005; Fry, Vogt, 1996; Oliveira, fev. 1996; Parés, 2006; Silveira, 2006; Slenes, fev. 1992; Souza, 2002.

${ }^{2}$ Ver Orser, Funari (1992). Pedro Funari, por sua vez, escreveu, ao longo dos últimos vinte anos, um grande número de artigos e capítulos de livros sobre a pesquisa arqueológica em Palmares. Um bom detalhamento das pesquisas, fornecendo informações sobre o campo e o material encontrado, está em Orser (1996).

${ }^{3}$ Projeto financiado pelo CNPq, processo número 472181/2011-4, sob a coordenação de Luís Cláudio Symanski e Flávio Gomes.

\section{REFERÊNCIAS}

ALLEN, Scott.

As vozes do passado e do presente: arqueologia, política cultural e o público na Serra da Barriga. Clio, Recife, v.20, n.1, p.81-101. (série Arqueológica). 2006.
BASTIDE, Roger.

As religiões africanas no Brasil: contribuição a uma sociologia das interpretações das civilizações. São Paulo: Pioneira. 1985. 
BASTIDE, Roger.

As Américas negras: as civilizações africanas no Novo Mundo. São Paulo: Difel/EdUSP. 1974.

CARLE, Cláudio.

A organização espacial dos assentamentos de ocupação tradicional de africanos e descendentes no Rio Grande do Sul, nos séculos XVIII e XIX. Tese (Doutorado) - Pontifícia Universidade Católica do Rio Grande do Sul, Porto Alegre. 2005.

CARNEIRO, Edison.

Ladinos e crioulos: estudos sobre o negro no Brasil. Rio de Janeiro: Civilização Brasileira. 1964.

CUNHA, Manuela Carneiro da.

Negros estrangeiros: os escravos libertos e sua volta à Africa. São Paulo: Brasiliense. 1985.

DANTAS, Beatriz Góis.

Vovô nagô e papai branco: usos e abusos da África no Brasil. Rio de Janeiro: Graal. 1982.

DAWDY, Shannon Lee.

Preface. Historical Archaeology, Rockville, v.34, n.3, p.1. 2000.

FARIA, Sheila.

A Colônia em movimento: fortuna e família no cotidiano colonial. Rio de Janeiro: Nova Fronteira. 1998.

FARIAS, Juliana Barreto; SOARES, Carlos Eugênio Líbano; GOMES, Flávio dos Santos. No labirinto das nações: africanos e identidades no Rio de Janeiro, século XIX. Rio de Janeiro: Arquivo Nacional. 2005.

FENNELL, Christopher.

Early African America: archaeological studies of significance and diversity. Journal of Archaeological Research, New York, v.19, n.1, p.1-49. 2011.

FERGUSON, Leland.

Uncommon ground: archaeology and early African America. Washington: Smithsonian Institution Press. 1992.

FRY, Peter; VOGT, Carlos.

Cafundó: a África no Brasil: linguagem e sociedade. São Paulo: Companhia das Letras. 1996.

GUIMARÃES, Carlos et al.

O quilombo do Ambrósio: lenda, documentos e arqueologia. Estudos Ibero-Americanos, Porto Alegre, v.16, n.1-2, p.161-174. 1990.

GUIMARÃES, Carlos; LANNA, Ana.

Arqueologia de quilombos em Minas Gerais.

Pesquisas - Antropologia, São Leopoldo, n.31, p.147-164. 1980.
GUNDAKER, Grey.

Discussion: creolization, complexity, and time. Historical Archaeology, Rockville, v.34, n.3, p.124-133. 2000.

HEINRICH, Adam.

Some comments on the archaeology of slave diets and the importance of taphonomy to historical faunal analyses. Journal of African Diaspora Archaeology and Heritage, Walnut Creek, v.1, n.1, p.9-40. 2012.

KLEIN, Herbert $S$.

American slavery in recent Brazilian scholarship, with emphasis on quantitative socio-economic studie. Slavery \& Abolition, London, v.30, n.1, p.111-133. 2009.

LARA, Silvia.

Campos da violência: escravos e senhores na Capitania do Rio de Janeiro, 1750-1808. Rio de Janeiro: Paz e Terra. 1988.

LEONE, Mark; FRY, Gladyz-Marie.

Spirit management among Americans of African descent. In: Orser, Charles (Org.). Race and the archaeology of identity. Salt Lake City: University of Utah Press. p.143-157. 2001.

LIMA, Tânia A., BRUNO, Maria Cristina O., FONSECA, Marta P.R.

Sintomas do modo de vida burguês no vale do Paraíba, século XIX: a fazenda São Fernando, Vassouras, RJ. Anais do Museu Paulista, São Paulo, v.1, n.1, p.170-206. 1993.

MACHADO, Noélia.; MILDER, Saul.

Negros e coronéis na região central do Rio Grande do Sul, um estudo de caso: o casarão dos Mello, Martinho da Serra. In: Encontro de Pós-graduação da Universidade do Vale do Paraíba, 3., 2006, São José dos Campos. Anais... São José dos Campos: Universidade do Vale do Paraíba. CD-ROM. 2006.

OLIVEIRA, Maria Inês Côrtes de.

Viver e morrer no meio dos seus. Revista USP, São Paulo, v.28, p.187-191. fev.1996.

ORSER, Charles.

A arqueologia de Palmares. In: Reis, João; Gomes, Flávio (Org.). Liberdade por um fio: história dos quilombos no Brasil. São Paulo: Companhia das Letras. p.26-51. 1996.

ORSER, Charles; FUNARI, Pedro.

A pesquisa arqueológica inicial em Palmares. Estudos Ibero-Americanos, Porto Alegre, v.18, n.2, p.53-69. 1992.

PARÉS, Luís Nicolau.

A formação do candomblé: história e ritual da nação jeje na Bahia. Campinas: EdUnicamp. 2006. 
RAMOS, Arthur.

As culturas negras no Novo Mundo. São Paulo: Companhia Editora Nacional. 1979.

RAMOS, Arthur.

O negro na civilização brasileira. Rio de Janeiro: Casa do Estudante do Brasil. 1953.

RAMOS, Arthur.

A aculturação negra no Brasil. São Paulo:

Companhia. Editora Nacional. 1942.

RAMOS, Arthur.

O negro brasileiro. Rio de Janeiro: Civilização Brasileira, 1935.

REIS, João José.

Rebelião escrava no Brasil: a história do levante dos malês em 1835. São Paulo: Companhia das Letras. 2003.

RODRIGUES, Nina.

Os africanos no Brasil. São Paulo: Companhia Editora Nacional. 1., ed., 1905. 1977.

SILVA, Eduardo.

Barões e escravidão: três gerações de fazendeiros e a crise da estrutura escravista. Rio de Janeiro: Nova Fronteira. 1984.

SILVEIRA, Renato da.

O candomblé da Barroquinha: processo de constituição do primeiro terreiro baiano de Keto. Salvador: Maianga. 2006.

SINGLETON, Teresa.

Cultural interaction and African American identity in plantation archaeology. In: Cusick, James (Org.). Studies in culture contact: interaction, culture change, and archaeology. Carbondale: Center for Archaeological Investigations. p.172-189. 1998.

SLENES, Robert W.

Da senzala uma flor: esperanças e recordações na formação da família escrava. Rio de Janeiro: Nova Fronteira. 1999.

SLENES, Robert W.

Malungu, Ngoma vem! África coberta e descoberta no Brasil. Revista USP, São Paulo, n.12, p.48-67. fev. 1992.

SOUZA, Marina de Mello.

Reis negros no Brasil escravista: história da festa de coroação de rei negro. Belo Horizonte: EdUFMG. 2002.
SOUZA, Marcos.

A vida escrava portas adentro: uma incursão às senzalas do Engenho de São Joaquim, Goiás, século XIX. Maracanan, Rio de Janeiro, v.7, p.83-109. 2011.

SOUZA, Marcos A., SYMANSKI, Luís.

Slave communities and pottery variability in Western Brazil: the plantations of Chapada dos Guimarães. International Journal of Historical Archaeology, New York, n.13, p.513-548. 2009.

SOUZA, Marcos A.; AGOSTINI, Camilla. Body marks, pots and pipes: some correlations between African scarifications and pottery decoration in eighteenth and nineteenthcentury Brazil. Historical Archaeology, Rockville, (no prelo).

STEIN, Stanley.

Vassouras: um município brasileiro do café (1850-1900). Rio de Janeiro: Nova Fronteira. 1990.

SYMANSKI, Luís.

O domínio da tática: práticas religiosas de origem africana nos engenhos de Chapada dos Guimarães (MT). Vestígios, Belo Horizonte, v.1, n.2, p.7-36. 2007.

SYMANSKI, Luís.

Slaves and planters in Western Brazil: material culture, identity and power. Doctoral Dissertation, University of Florida, Gainesville. 2006.

SYMANSKI, Luís; SOUZA, Marcos A. A arqueologia histórica: relações sociais e construção de identidades na região do rio Manso, séculos XVIII e XIX. In: Fraga, Leila (Org.). História e antropologia no vale do rio Manso. Goiânia: EdUCG. p.241-264. 2006.

TAVARES, Áurea Conceição Pereira. Vestígios materiais nos enterramentos na antiga Sé de Salvador: postura das instituições religiosas africanas frente à Igreja católica em Salvador no período escravista. Dissertação (Mestrado) Departamento de História, Universidade Federal de Pernambuco, Recife. 2006.

WILKIE, Laurie.

Magic and empowerment on the plantation. Southeastern Archaeology, s.l., v.14 n.2, p.136-157. 1995.

\section{$\rightarrow \rightarrow \rightarrow<<$}

